\title{
Bilan de masse et érosion dans le bassin de l'Oued Mina Nord-ouest algérien
}

\author{
Faiza Hallouz ${ }^{1,2}$, Abdelhadi Ammari ${ }^{2}$, Mohamed Meddi ${ }^{2}$, Ouarda Yasmine Nait Yahia ${ }^{2}$, and \\ Ali Rahmani Salah Eddine ${ }^{3}$ \\ ${ }^{1}$ University of Khemis Miliana, 44225 Ain Defla, Algeria \\ ${ }^{2}$ LGEE, École Nationale Supérieur d'Hydraulique, BP 31, 09000 Blida, Algeria \\ ${ }^{3}$ Geo-Environment Laboratory, Faculty of Earth Sciences, Geography and Spatial Planning, University of \\ Sciences and Technology Houari Boumediene, 16111 Bab Ezzouar Algiers, Algeria \\ Correspondence: Faiza Hallouz (f.hallouz@univ-dbkm.dz)
}

Published: 16 November 2021

\begin{abstract}
Résumé. Le taux de sédimentation des barrages du bassin hydrographique du Cheliff reste le plus élevé de toutes les régions de l'Algérie. L'oued Mina est un affluent majeur de l'oued Cheliff, le plus grand oued de l'Algérie qui transporte un taux de sédiment important et le barrage de Sidi M'Hamed Ben Aouda (Relizane) qui barre cet oued a reçu un volume de vase égal à 6,7 millions $\mathrm{de}^{3}$ par année. L'objectif de cette étude est de proposer un modèle d'estimation de l'érosion spécifique permettant de construire un ensemble d'indicateurs afin d'identifier les zones prioritaires nécessitant le renforcement des moyens de lutte contre l'érosion et une exploitation meilleure des barrages qui sont la seule ressource pour l'irrigation et l'activité agricole de la région en prolongeant leur durée de vie. Nous avons procédé à l'analyse des données journalières de pluie et de débits des stations hydro-pluviométriques d'oued El Abtal (de 1985-2010) du sous bassin versant dont les apports s'accumulent dans le barrage SMBA. Ce travail nous a permis d'élaborer une carte de sensibilisation qui délimite les zones vulnérables selon le taux d'érosion spécifique (Es oued Haddad=18.3, Es oued El Abd=7.3, Es oued Mina $=10.47\left(\mathrm{tha}^{-1} \mathrm{an}^{-1}\right) \ldots$ calculés à partir du modèle trouvé dans cette étude. Enfin, l'état érosif des versants a été divisé en quatre classes principales de vulnérabilité des sols, à savoir : les niveaux «négligeable », «modéré », «élevé » et « très élevé ». Cette carte permet de faciliter la prise de décisions relatives à la priorisation des zones à préserver ou à restaurer.
\end{abstract}

\section{Introduction}

Depuis des siècles, les géographes ont considéré la zone des bassins méditerranéens comme très sensible à l'érosion sous toutes ses formes (Roose et al., 2012). L'Algérie, faisant partie de cette zone, n'est pas à l'abri de ce phénomène, il est parmi les pays les plus menacés par l'érosion hydrique (Achite et al., 2006).

En effet, en Algérie, le taux d'érosion spécifique atteint les valeurs les plus importantes d'Afrique du Nord, dépassant les $2000 \mathrm{t} \mathrm{km}^{-2} \mathrm{an}^{-1}$ et concerne la plupart des bassins versants de l'Atlas tellien (Rhiou, Sly, Fodda, Mazafran, Isser, Soummam...), il atteint $4000 \mathrm{t} \mathrm{km}^{-2} \mathrm{an}^{-1}$ sur la chaîne des fleuves côtiers Dahra et $5000 \mathrm{t} \mathrm{km}^{-2} \mathrm{an}^{-1}$ sur le bassin
d'Ighil-Emda (Demmak, 1982). L'Algérie est de ce fait l'un des pays les plus menacés dans le monde par l'érosion.

L'envasement est la conséquence directe de l'érosion et la sédimentation, qui sont aussi la cause de la dégradation des sols agricoles ; $45 \%$ des sols agricoles du Nord Algérien sont directement menacés par l'érosion, soit 12 Millions d'hectares (Chebbani et al., 1999).

Le taux de sédimentation des barrages du bassin hydrographique du Cheliff reste le plus élevé de toutes les régions de l'Algérie (Remini, 2006). L'oued Mina est un affluent majeur de l'oued Cheliff, le plus grand oued de l'Algérie qui transporte un taux de sédiment important. A titre d'exemple, le barrage de Sidi M'Hamed Ben Aouda (Relizane) qui barre cet oued a reçu un volume de vase égal à 6,7 millions de $\mathrm{m}^{3}$ 


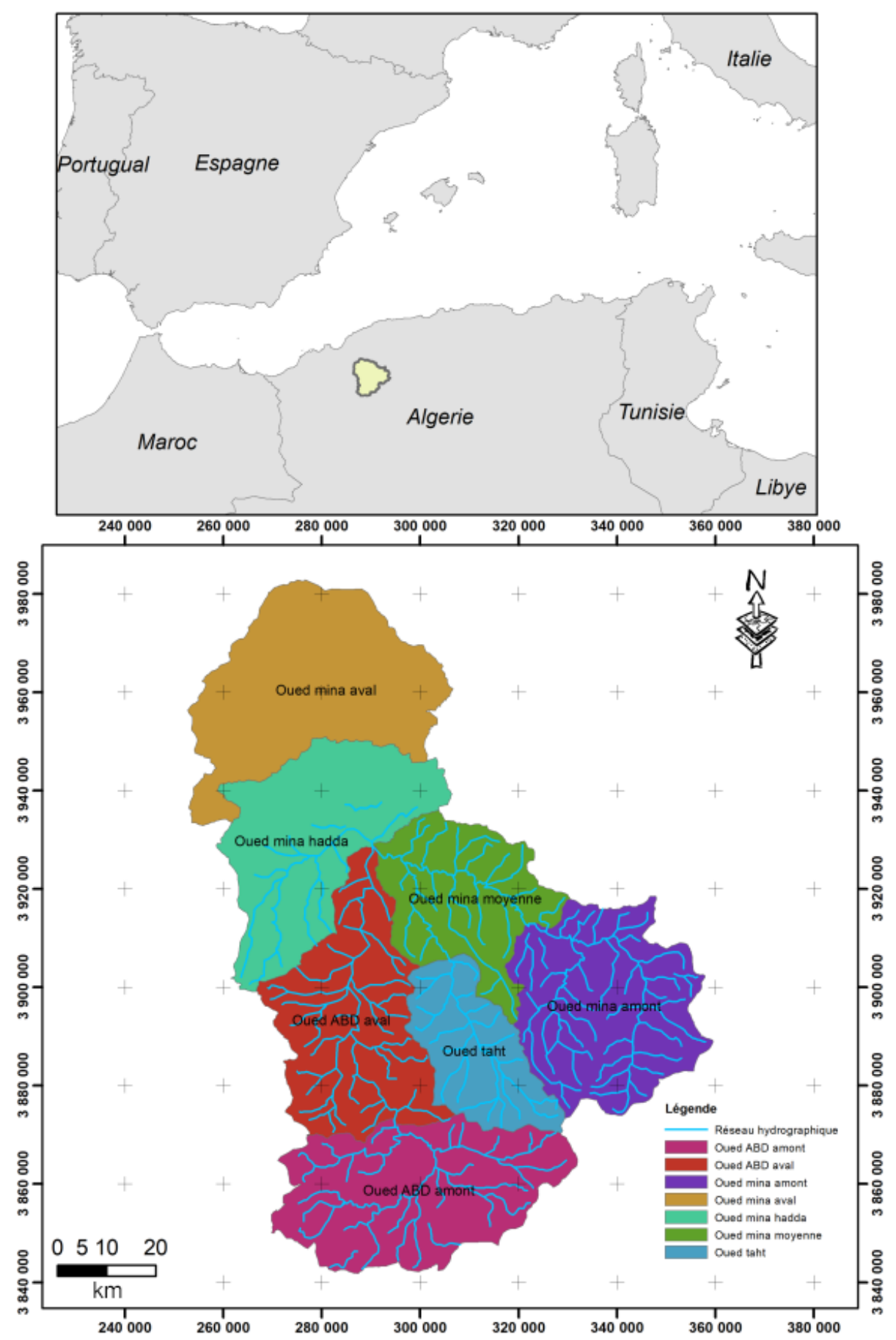

Figure 1. Bassin versant de l'oued Mina.

par année durant la période 1995-2003 (Remini et Bensafia, 2012; Meddi, 1992; Kouri, 1993; Touaïbia, 2000; Achite, 2002; Hallouz, 2013; Toumi, 2013).

La maîtrise de ce phénomène ne peut passer qu'al̀ travers des modèles qui nous permettent de décrire et de quantifier dans la mesure du possible les taux de dégradation des sols et ainsi les conséquences qui se suivent. La validitêi de ces mo- dèles est tributaire de la qualité et la quantité des données disponibles. Le manque de données conduit souvent l'ingénieur à utiliser les méthodes empiriques disponibles afin d'évaluer les quantités de sédiments transportés annuellement par les cours d'eau (Meddi et al., 1998), sans que nous nous ayons des dispositifs de contrôle de ces résultats. 
Le principal objectif de ce travail est de proposer un modèle d'estimation de l'érosion spécifique permettant de construire un ou un ensemble d'indicateurs permettant d'identifier les zones prioritaires nécessitant le renforcement des moyens de lutte contre l'érosion et déterminant des scénarios pour une gestion de l'occupation et l'utilisation du sol à long terme dans le but de minimiser l'érosion hydrique et une exploitation meilleure des retenues et des barrages qui sont la seule ressource pour l'irrigation et l'activité agricole de la région en prolongeant leur durée de vie. Nous tenterons de mettre en évidence les risques directs d'envasement des barrages situés dans le bassin de l'oued Mina, des risques liés directement al la cadence accélérée de l'érosion hydrique, avec la dégradation continue du couvert végétal comme facteur principal.

Vue le changement continue du cycle hydrologique et du climat de la région, le modèle le plus représentatif est certainement celui baseì sur les données les plus récentes, malheureusement, la décennie noire qu'a connue l'Algérie a eu son impact sur la collecte des données, pour cela, nous nous sommes intéressés à la mesure hydrologique, en proposant des méthodes simples et précises, qui permettrons sans doute de pallier au manque de données hydrométriques et du transport solide, en augmentant facilement la fréquence des mesures avec une meilleur qualité de la donnée.

Nous proposons à la fin de cette étude une approche cartographique de la variation de l'érosion hydrique afin de prévoir le risque érosif et prendre les précautions nécessaires de lutte contre les dégâts causés au niveau des barrages et des surfaces du sol.

\section{Zone d'étude, données et méthodes}

\subsection{Présentation de la zone d'étude}

Situé en zone semi-aride, le bassin versant de l'Oued Mina répond à notre objectif en raison de sa forte érodabilité et de la disponibilité des données pluviométriques et hydrométriques. Situé à environ $300 \mathrm{~km}$ à l'ouest d'Alger, entre $0^{\circ} 20^{\prime}$ et $1^{\circ} 10^{\prime} \mathrm{E}$ et entre $34^{\circ} 40^{\prime}$ et $35^{\circ} 40^{\prime} \mathrm{N}$, le bassin versant de l'Oued Mina draine, au niveau du Barrage de Sidi M'Hamed Benaouda (SMBA), une superficie de $6000 \mathrm{~km}^{2}$ (Fig. 1). L'Oued Mina est parmi les principaux affluents de l'Oued Cheliff. Il parcourt une distance de $90 \mathrm{~km}$ environ entre les barrages de Bakhada et de Sidi M'Hamed Benaouda avec une orientation Sud Est-Nord-Ouest.

Le climat de la région est de type semi-aride méditerranéen avec une précipitation moyenne annuelle de $305 \mathrm{~mm}$ marquée par une irrégularité aussi bien saisonnière qu'interannuelle (Meddi, 1992). Par ailleurs, l'analyse de la répartition géographique et de la diversité des formations végétales sur le bassin a montré deux parties nettement distinctes (Mahieddine, 1997; Kouri, 1993) : la zone Nord purement marneuse, fortement érodée et dépourvue de végétation à l'exception de quelques îlots de reboisement et de plantations

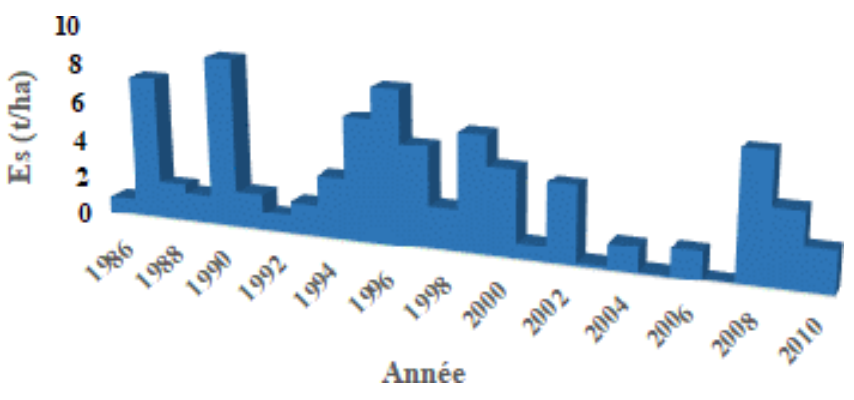

Figure 2. Dégradation spécifique dans le bassin de l’Oued Mina.

d'arbres fruitiers dans la vallée; et la zone sud moins érodée et dont environ $50 \%$ de la surface sont recouverts par une végétation de densité très variable et très parsemée, allant de la forêt (Pin d'Alep) localisée au maquis très éparse. Il y a lieu de noter qu'une agriculture d'autosubsistance y règne avec une surexploitation des sols, un défrichement permanent et un surpâturage intensif.

\subsection{Acquisition et préparation des données}

Dans cette étude nous avons utilisé les données journalières de pluies et de débits de la station d'Oued El Abtal sur une période allant de 1985 à 2010 (25 années). Ces données proviennent de la base de données de l'Agence Nationale des Ressources Hydrauliques (ANRH, 2010). Le débit liquide moyen interannuel dans ce bassin est égal à $2,44 \mathrm{~m}^{3} / \mathrm{s}$ avec un coefficient de variation de 0.44 et une pluie moyenne annuelle de l'ordre de $430 \mathrm{~mm}$ (ANRH, 2010).

\subsection{Estimation de la dégradation spécifique}

La dégradation spécifique ou l'érosion spécifique (ES) donne une idée sur l'érodabilité de la région, et donc sur la vulnérabilité à l'envasement des ouvrages de stockage existants. L'érosion spécifique moyenne interannuelle est le rapport entre l'apport solide moyen interannuel et la superficie du bassin drainé (Touainia et Achite, 2003).

Es $=\frac{\mathrm{As}}{S}$ en $\left[\mathrm{tkm}^{-2} \mathrm{an}^{-1}\right]$ ou $\left[\mathrm{tha}^{-1} \mathrm{an}^{-1}\right]$

Avec : As : Apport solide moyen interannuel $\left[\mathrm{t} \mathrm{km}^{-2}\right], S$ : Superficie du bassin drainé $\left[\mathrm{km}^{2}\right]$.

\subsection{Modélisation du transport solide}

Afin de générer un modèle représentatif de l'érosion dans notre zone d'étude qui est d'une superficie de plus de $6000 \mathrm{~km}^{2}$ en utilisant des indicateurs en rapport avec les conditions sur site, nous avons procédé à une analyse multivariée, avec des régressions multiples, de l'érosion spécifique en fonction de divers paramètres (climatiques, hydro 


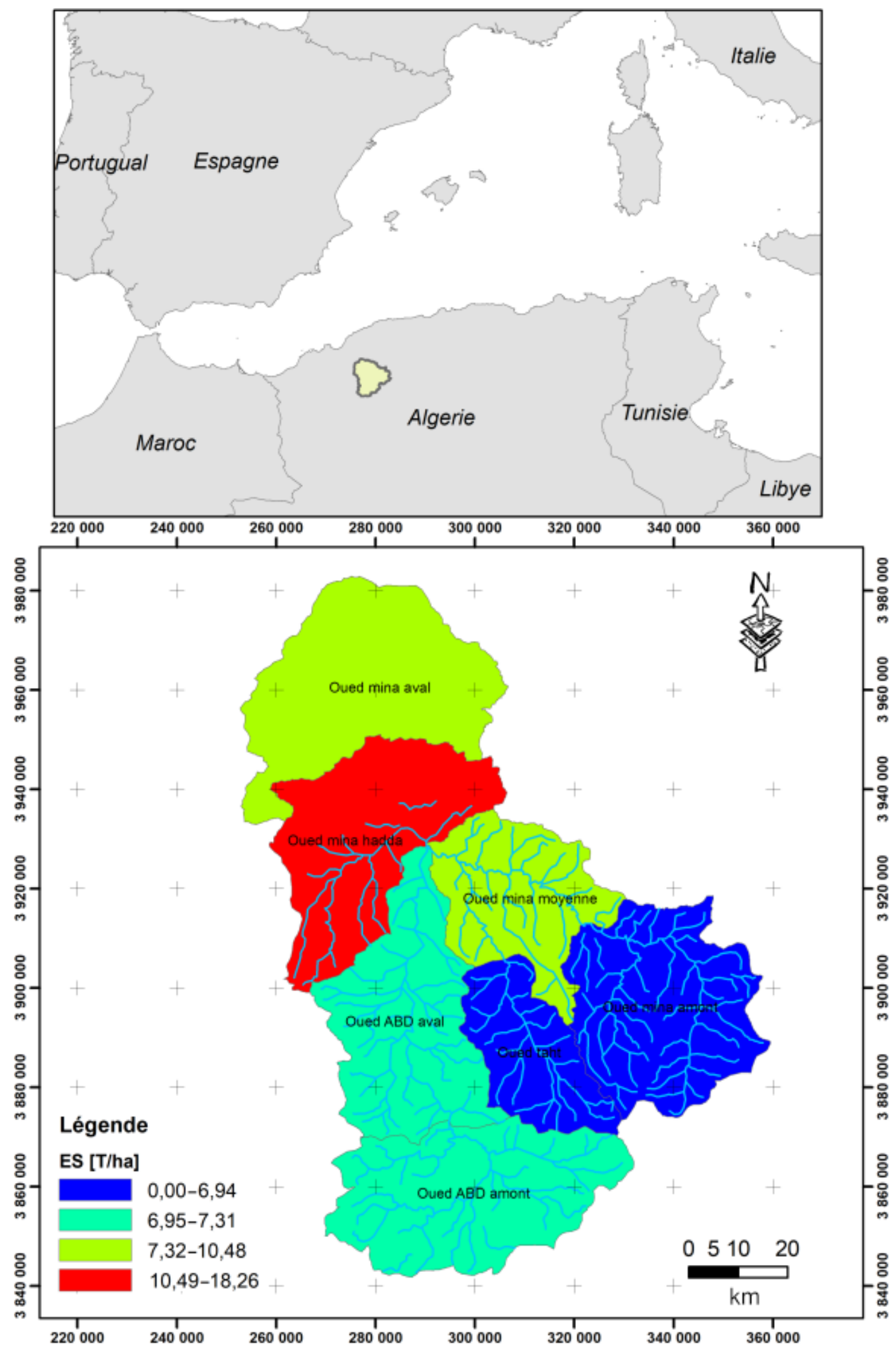

Figure 3. Évolution de l'érosion spécifique dans le bassin versant de l'Oued Mina. 
Tableau 1. Récapitulatif des bassins versants.

\begin{tabular}{lrrrrrrrrrrrrr}
\hline Sous bassins & $S$ & $P$ & $\mathrm{Ic}$ & $L$ & $l$ & $L_{\mathrm{TP}}$ & $D$ & $\mathrm{Ds}$ & $\mathrm{Ig}$ & $\mathrm{Le}$ & $\mathrm{Dd}$ & $\mathrm{Ct}$ & $\mathrm{Tc}$ \\
\hline Oued Haddad & 470 & 100 & 1.29 & 36.95 & 12.58 & 47 & 935 & 542 & 1.84 & 23.71 & 0.53 & 0.05 & 10.1 \\
Oued EL ABTAL & 5365 & 256 & 1.45 & 108.3 & 40.54 & 120 & 660 & 312 & 0.61 & 23.12 & 0.45 & 0.05 & 17.4 \\
Oued Taht & 852 & 153 & 1.46 & 62.9 & 19.78 & 67.5 & 660 & 307 & 1.05 & 16.70 & 0.46 & 0.03 & 10.4 \\
Oued Mina & 2190 & 275 & 1.64 & 119.1 & 41.3 & 130 & 870 & 342 & 0.73 & 14.77 & 0.4 & 0.04 & 17.5 \\
Au droit du barrage SMBA & 6132 & 352 & 1.26 & 128.5 & 47.73 & 135 & 1194 & 728 & 0.6 & 14.52 & 0.96 & 0.08 & 23.5 \\
\hline
\end{tabular}

On a rassemblé tous les facteurs susceptibles d'influencer le phénomène à savoir (Ammari, 2012) : La superficie des sous bassins $(S)\left(\mathrm{km}^{2}\right)$. La précipitation moyenne interannuelle $(P)(\mathrm{mm})$. L'indice de compacité $(\mathrm{Ic}) . L$ : longueur du rectangle équivalent $(\mathrm{km}) . l$ : largeur du rectangle équivalent $(\mathrm{km})$. Longueur du talweg principal $(L \mathrm{TP})$ $(\mathrm{km})$. Altitude moyenne $(D)(\mathrm{m})$. Dénivelée spécifique $(\mathrm{Ds})(\mathrm{m})$. L'indice de pente globale (Ig). La lame écoulée moyenne interannuelle (Le) (cm). La densité de drainage (Dd) $\left(\mathrm{km} / \mathrm{km}^{2}\right)$. Le coefficient de torrentialité $(\mathrm{Ct})$. Temps de concentration (Tc) (h).

Tableau 2. Matrice de corrélation.

\begin{tabular}{|c|c|c|c|c|c|c|c|c|c|}
\hline Variable & $\begin{array}{r}\text { Es } \\
\left(\mathrm{tha}^{-1} \mathrm{an}^{-1}\right)\end{array}$ & $\begin{array}{r}S \\
\left(\mathrm{~km}^{2}\right)\end{array}$ & Ic & $\begin{array}{r}\text { Dd } \\
\left(\mathrm{km} \mathrm{km}^{-2}\right)\end{array}$ & Ig & $\mathrm{Ct}$ & $\begin{array}{r}\text { Le } \\
(\mathrm{cm})\end{array}$ & $\begin{array}{r}P \\
(\mathrm{~cm})\end{array}$ & $\begin{array}{r}\mathrm{Ce} \\
(\%)\end{array}$ \\
\hline Es & 1,00 & & & & & & & & \\
\hline$S$ & 0,31 & 1,00 & & & & & & & \\
\hline Ic & $-0,77$ & $-0,01$ & 1,00 & & & & & & \\
\hline Dd & 0,49 & 0,58 & 0,16 & 1,00 & & & & & \\
\hline Ig & $-0,03$ & $-0,90$ & 0,00 & $-0,17$ & 1,00 & & & & \\
\hline $\mathrm{Ct}$ & 0,83 & 0,70 & $-0,66$ & 0,49 & $-0,52$ & 1,00 & & & \\
\hline $\mathrm{Le}$ & 0,01 & 0,58 & 0,42 & 0,47 & $-0,44$ & 0,04 & 1,00 & & \\
\hline$P$ & $-0,22$ & 0,38 & $-0,13$ & $-0,51$ & $-0,70$ & 0,09 & 0,28 & 1,00 & \\
\hline $\mathrm{Ce}$ & 0,42 & 0,68 & 0,03 & 0,57 & $-0,46$ & 0,40 & 0,91 & 0,24 & 1,00 \\
\hline
\end{tabular}

Es : Érosion spécifique moyenne interannuelle $\left(\mathrm{tha}^{-1} \mathrm{an}^{-1}\right) . S:$ La superficie des sous bassins $\left(\mathrm{km}^{2}\right)$. Ic : L'indice de compacité. Dd : La densité de drainage $\left(\mathrm{km} / \mathrm{km}^{2}\right)$. Ig : L'indice de pente globale. Ct : Le coefficient de torrentialité. Le : La lame écoulée moyenne interannuelle $(\mathrm{cm}) . P$ : La précipitation moyenne interannuelle $(\mathrm{cm})$. Ce : Le coefficient d'écoulement.

morpho-métriques ...) contenus dans le Tableau 1. Ces regressions ont e'te' calcule'es en utilisant le logiciel statistique XL-STAT (2013).

\section{Résultats et discussion}

\subsection{Estimation de la dégradation spécifique}

Dans le bassin versant de l'oued Mina, on estime une dégradation spécifique moyenne interannuelle de l'ordre de 4,4 $\mathrm{tha}^{-1} \mathrm{an}^{-1}$ (Fig. 2).

Les valeurs de l'érosion spécifique calculées sont expliquées par la nature du relief, la géologie ainsi que la nature des cours d'eau dans le bassin versant; les dépôts alluvionnaires et les lâchées du barrage Bakhadda favorisent également le phénomène. Le couvert végétal contribue énormément à la réduction de l'action érosive aussi.

Toutefois, on constate que ces valeurs concordent avec un certain nombre de travaux d'aménagement de la région d'étude sur les différents niveaux (Meddi, 1992; Touaibia et Achite, 2003; Hallouz et al., 2012, 2018).

\subsection{Modélisation du transport solide}

La matrice des coefficients de corrélation est illustrée dans le Tableau 2.

La sélection finale des paramètres est basée sur l'étude de régression multiple.Nous utilisons le logarithme du paramètre pour linéariser le modèle.

Ainsi, le modèle le plus représentatif est :

Es $=e^{8.93} L_{e}{ }^{0.83} C_{t}{ }^{0.87} D_{d}{ }^{0.23} S^{-0.87}$

Avec des coefficients de régression multiple et partielle proche de 1 .

Le test de Student a démontré la significativité du coefficient le plus petit au seuil de $5 \%$ comme le suggère Laborde (2003) car il a moins de $5 \%$ de chance qu'il est dû au hasard.

Sur la table de Fisher - Snedecor, pour un seuil de confiance de $5 \%$ et pour des degrés de liberté de $(p-1)$ et $(n-p+1): F_{0,95}=9,55<F_{\mathrm{obs}}$. D'après le résultat obtenu, on peut dire que le modèle est significatif.

Après avoir développé un modèle de calcul de l'érosion spécifique avec analyse multivariée, une cartographie de la vulnérabilité à l'érosion hydrique a été réalisée (Fig. 3). La méthode est basée sur des données saisies sur SIG (QGis), 
en représentant l'érosion des sols de l'ensemble de la zone d'étude.

Ce support cartographique nous permet de visualiser les différentes zones à risque érosif sur l'ensemble du bassin de la Mina. Ce dernier partagé en quatre sous bassin versant et ainsi partagé en quatre régions suivant le degré d'érosion dans chacune d'elle.

On remarque un faible risque dans la région sud-ouest (oued Abd) et encore moins en est (oued Mina amont et oued Taht) car la région est caractérisée par des formations géologiques et lithologique denses et consolidées. Tandis que le risque augmente en allant vers le haut (vers le Nord) appelée Oued Haddad situé en amont du barrage SMBA ce qui explique clairement le risque d'envasement du réservoir de cet ouvrage hydraulique.

Enfin, cette étude nous a permis d'aboutir à des indicateurs permettant d'identifier les zones prioritaires (Fig. 3) nécessitant le renforcement des moyens de lutte contre l'érosion et déterminant des scénarios pour une gestion de l'occupation et l'utilisation du sol à long terme dans le but de minimiser l'érosion hydrique et une exploitation meilleure des retenues et des barrages qui sont la seule ressource pour l'irrigation et l'activité agricole de la région en prolongeant leur durée de vie.

\section{Conclusions}

En Algérie, l'érosion hydrique est un problème majeur. Elle devient dangereuse dès qu'elle dépasse le seuil tolérable et son intensitéì varie d'une zone aÌ l'autre.

L'objectif de ce travail est la mise au point d'une méthode d'estimation de l'érosion spécifique qui permettra aux gestionnaires des ouvrages et au projeteurs de projet de mieux prédire ce phénomène et ainsi prédéterminer l'importance et la nature des ouvrages et les pertes de capacité possible suite à l'envasement; les résultats estimés devraient être confrontés aux observations et au mesures lors de l'études pour un meilleur choix.

Par ailleurs, nous avons analysé des données journalières de pluie et de débits des stations hydro-pluviométriques d'oued El Abtal (1985-2010) du sous bassin versant dont les apports s'accumulent dans le barrage SMBA.

Un modèle basé sur une analyse multivariée de divers facteurs climatiques, hydro morpho- métriques nous a permis d'aboutir à des indicateurs influençant les scénarios du risque érosif sur le bassin versant. Ce travail a permis d'élaborer une carte de sensibilisation qui délimite les zones vulnérables selon le taux d'érosion spécifique (Es oued Haddad $=18.3$, Es oued El Abd=7.3, Es oued Mina $=10.47\left(\mathrm{tha} \mathrm{an}^{-1}\right)$ ... calculés à partir du modèle trouvé.

Enfin, le risque d'érosion augmente en allant vers le haut (vers le Nord) sous bassin de l'Oued Haddad situé en amont du barrage SMBA ce qui explique clairement le risque d'envasement du réservoir de cet ouvrage hydraulique.
Disponibilité du code. Le code du logiciel XL-STAT qui a été utilisé dans cette étude peut être trouvé à l'adresse suivante : XLSTAT : id de la license est 0117590, version du 04.02.2013 (Addinsoft, 2014).

Collaborateurs. FH wrote the main paper, and FH, AA, MM, OYNY, ARSE discussed the results and implications and commented on the manuscript at all stages. FH, AA, MM, OYNY, ARSE contributed extensively to the work presented in this paper.

Intérêts concurrents. Les auteurs déclarent qu'ils n'ont aucun conflit d'intérêts.

Clause de non-responsabilité. Publisher's note : Copernicus Publications remains neutral with regard to jurisdictional claims in published maps and institutional affiliations.

Déclaration du numéro spécial. This article is part of the special issue "Hydrology of Large River Basins of Africa". It is a result of the 4th International Conference on the "Hydrology of the Great Rivers of Africa”, Cotonou, Benin, 13-20 November 2021.

Remerciements. Merci a' l'Agence Nationale des Ressources Hydrauliques (ANRH) pour les donne'es hydrome'triques et pluviome triques. Ce travail a e'te' re'alise' dans le cadre du projet SICMED Indicateurs, composante de MISTRALS SICMED, INSU CNRS.

\section{Références}

Achite, M. : Approche statistique d'e'valuation du transport solide dans le bassin versant de l'oued Mina (nord ouest Alge'rien), Colloque international sur l'eau dans le bassin me'diterrane'en : Ressources et de'veloppement durable, 10-13 octobre 2002, Monastir (Tunisie), 894-899, 2002.

Achite, M., Touaibia, B., and Ouillon, S. : 'Érosion hydrique en Algérie du Nord : Ampleur, Conséquences and Perspectives «Haddad (Nord-Ouest algérien) » 14th International Soil Conservation Organization Conference, Water Management and Soil Conservation in Semi-Arid Environments. Marrakech, Morocco, 14-19 May 2006, 2006.

Addinsoft : XLSTAT, Analyse de données et statistique avec MS Excel, Addinsoft, NY, USA, 2014.

ANRH (Agence Nationale des Ressources Hydrauliques) : Les changements climatiques and leur impact sur les ressources en eau en Algérie, in : Proceedings of the Assises Nationales sur L'Eau, Fez, Morocco, 25-29 October 2010, ANRH, Alger, Algeria, 41 pp., 2010.

Chebbani, R., Djilli, K., et Roose, E. : Étude des risques d'eìrosion dans le bassin versant de l'Isser, Algérie, Bulletin Réseau Érosion, 19, 85-95, 1999. 
Demmak, A. : Contribution à l'étude de l'érosion et des Transports Solides en Algérie Septentrionale, Ph.D. Thesis, Université de Pierre et Marie Curie, Paris, France, 1982.

Hallouz, F. : Transport Solide Dans le Bassin D'El Oued Mina et Sédimentation du Barrage SMBA, Ph.D. Thesis, Es-Sciences, École Nationale Supérieure d'Hydraulique (ENSH), Soumaâ, Algeria, 219 pp., 2013.

Hallouz, F., Meddi, M., et Mahé, G. : Relation débit liquidetransport de matières en suspension dans le bassin de l'Oued Mina, en amont du barrage Sidi M'Hamed Ben Aouda (Oued Cheliff), Nord-Ouest d'Algérie, 1ère Conférence internationale IS-RIVERS, Recherches et actions au service des fleuves et grandes rivières, Lyon, France, 2012.

Hallouz, F., Meddi, M., Mahé, G., Toumi, S., and Rahmani, S. E. A. : Erosion, Suspended Sediment Transport and Sedimentation on the Wadi Mina at the Sidi M'Hamed Ben Aouda Dam, Algeria, Water, 2018, 10, 895, 31 pp., https://doi.org/10.3390/w10070895, 2018.

Kouri, L. : L'e'rosion hydrique des sols dans le bassin versant de l'Oued Mina. Etude des processus et type de fonctionnement des ravins dans la zone des marnes tertiaires, The`se de doctorat, Universite’ Louis Pasteur, Strasbourg, France, 238 pp., 1993.

Mahieddine, M. : Quantification and Variabilité Parcellaire Sous Simulation de Pluie Dans le Bassin Versant de L'Oued Mina, PhD. Thesis, École Nationale Supérieure des Sciences Agronomiques, Alger, Algeria, 120 pp., 1997.
Meddi, M. : Hydro-pluviométrie et transport solide dans le bassin versant de l'oued Mina (Algérie). Thèse de doctorat, Université, Louis Pasteur, Strasbourg, France, 285 pp., 1992.

Remini, B. : Valorisation de la vase des barrages - quelques exemples algériens, Larhyss Journal, No. 05, 75-89, Juin 2006.

Remini, B. et Bensafia, D. : Envasement du Barrage de SMBA Algérie. Comité Scientifique Projet SIGMED Rabat Maroc, 2012.

Roose, É., Sabir, M., Arabi, M., Morsli, B., et Mazour, M. : Soixante années de recherches en coopération sur l'érosion hydrique et la lutte antiérosive au Maghreb, Physio-Géo [En ligne], Vol. 6, mis en ligne le 3 mai 2012, 43-69, https://doi.org/10.4000/physiogeo.2319, 2012.

Touaïbia, B. et Achite, M. : Contribution à la cartographie de l'érosion spécifique du bassin versant de l'Oued Mina en zone semiaride de l'Algérie septentrionale, Hydrol. Sci. J., 48, 235-242, 2003.

Toumi, S. : Application des Techniques Nucléaires et de la Télédétection à L'étude de L'érosion Hydrique Dans le Bassin Versant de L'oued Mina, Ph.D. Thesis, Es-Sciences, École Nationale Supérieure d'Hydraulique (ENSH), Soumaâ, Algeria, 189 pp., 2013. 